\title{
DNA REPAIR MECHANISMS AND CELLULAR REPROGRAMMING: CRITERIA FOR THE SUCCESSFUL GENERATION OF HUMAN INDUCED PLURIPOTENT STEM CELLS AND IN VITRO DISEASE MODELLING
}

\author{
Lyle Armstrong ${ }^{1}$, Majlinda Lako ${ }^{1}$ and Miodrag Stojkovic ${ }^{2,3}$ \\ Institute of Genetic Medicine, Newcastle University, Newcastle NE1 3BZ, UK \\ ${ }^{2}$ Human Genetics Department, Faculty of Medical Sciences, University of Kragujevac, Serbia \\ ${ }^{3}$ SPEBO MEDICAL, Leskovac, Serbia
}

\begin{abstract}
Maintenance of genomic integrity is an important aspect of stem cell function

All stem cells, whether they are pluripotent or multipotent tissue-specific cells, need to be able to eliminate genomic mutations if they threaten the ability of the cell to complete its function in vivo. This is of great importance for adult stem cells, the purpose of which is the lifelong repair and regeneration of a specific organ system. It is easy to imagine the potential harm that could accumulate in the haematopoietic system if only a small fraction of the haematopoietic stem cells (HSCs) resident in the endosteal niche of the bone marrow (BM) were allowed to accumulate mutations that altered their ability to generate lymphoid or myeloid lineages. Because the daily requirement
\end{abstract}

\section{PLURIPOTENT STEM CELLS SHARE THE NECESSITY OF MAINTAINING GENOME INTEGRITY}

Human pluripotent stem cells is the common term used to describe two types of pluripotent stem cells characterised by an indefinite self-renewal ability and the capacity to give rise to any cell type in the adult: human embryonic stem cells (hESCs) and human induced pluripotent stem cells (hiPSCs). hESCs are derived from spare in vitro fertilised embryos (1) after parental consent and have been widely used in the last decade as a generic tool to understand the maintenance of pluripotency, human embryonic development, and congenital disease. However, the utilisation of human embryos for research purposes is surrounded by a number of ethical issues, prohibiting hESC derivation and application in several countries. The main practical issue related to their application is the evidence that differentiated progeny cells express human leukocyte antigens (HLAs) that will most likely result in graft rejec- for new blood cells is of the order of one billion, damage of this type would soon become apparent, and the patient's health would decline. Important though this is, the impact of mutations occurring in adult life is minor compared to the possible disruptions that could arise if the genome of the early embryo was damaged to the point that embryonic development was restricted. The inner cell mass (ICM) of blastocyst-stage embryos gives rise to every tissue found in the adult but consists of a very small number of cells (which can be as few as 12 cells). If a mutation is allowed to persist in any of the ICM cells, it will be transferred to the tissues and organs that arise from that cell and could have a profound effect on organ function. Therefore, the accumulation of mutations in the ICM must be prevented at all costs.

Keywords: pluripotent stem cells, DNA damage, DNA repair

tion and could be bypassed only by the creation of HLAtyped hESC banks from which a best match can be selected. Human iPSCs bypass both of these issues because they are generated by the reprogramming of somatic cells back to the pluripotent state, akin to embryonic stem cells (2-4). As such, these cells share all the characteristics of hESCs, including the ability to proliferate indefinitely and differentiation into many cell types, but also represent a source of autologous stem cells for therapies, provided that they are derived from the patient in which they are used. Such patient-derived cells present a unique opportunity to create in vitro disease models that can be exploited to understand disease pathology and drug discovery (see Figure 1).

The enhanced genome defence abilities of pluripotent stem cells decrease markedly upon differentiation (5-7). A number of studies have demonstrated that such terminally differentiated cell types as neurons differ greatly in their DNA repair capacity and ability to remove potentially harmful reactive oxygen species, such as superoxides and peroxides $(8,9)$. Because the other publications in this field have dealt adequately with 


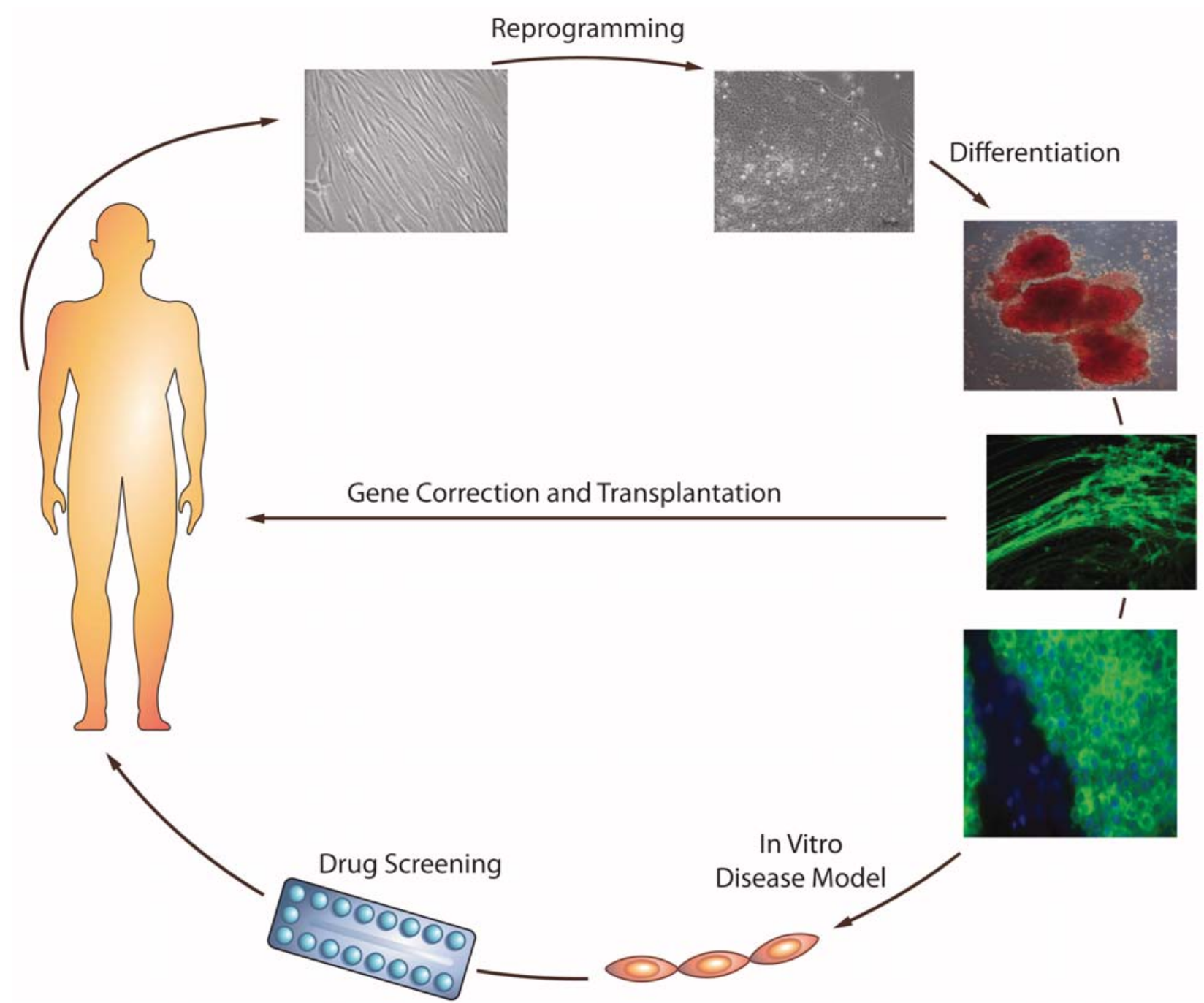

Figure 1. Schematic presentations of iPSC technology and its applications for basic biology, drug discovery and cell replacement therapies.

the mechanisms of such defence systems, these mechanisms will not be discussed in detail here; however, it is apparent that the decrease in genome defence activity that accompanies the differentiation of pluripotent stem cells must be reversed when somatic cells re-acquire pluripotency during hiPSC generation. This reprogramming of genome defence has been demonstrated in a few recent studies showing that the expression levels of DNA repair-related genes were equivalent in hiPSCs and hESCs and were higher in comparison with their differentiated counterparts (10). At a first glance, these data could be considered to simply underline the point that pluripotent cells require efficient DNA repair; however, other studies suggest a more fundamental relationship between pluripotency and genome defence. The pluripotent state depends upon a framework of transcription factors that are largely controlled by the "core" factors OCT4, SOX2 and NANOG (11). These factors, in turn, are subjected to regulation by several "activator" protein complexes; among these, the XPC-nucleotide excision repair complex (12) has been shown to interact with both the OCT4 and SOX2 gene products and is recruited to the NANOG and OCT4 promoters. Importantly, the selective depletion of the components of the XPC complex using siRNA directed against
$X P C, R A D 23 B$ and CETN2 caused hESCs to differentiate, suggesting that, although pluripotent cells have a fundamental need for enhanced DNA repair, the expression of the genes involved in DNA repair at an appropriate level is also central to the maintenance of pluripotency (12).

If pluripotency relies, at least in part, on effective DNA repair, it is then possible that DNA repair processes contribute to the reprogramming of somatic cells into hiPSCs. The reprogramming process is very inefficient; typically less than $1 \%$ of the transduced somatic cells give rise to truly pluripotent colonies. However, p53-null mouse embryonic fibroblasts reprogramme much more efficiently than do their wildtype counterparts. p53 is an important protein in cell cycle control and the initiation of DNA repair in cells with damaged genomes (13); more importantly, it prevents the propagation of such DNAdamaged cells (14). In view of this fact, it has been suggested that p53 may prevent the reprogramming of cells with DNA damage or chromosomal abnormalities from progressing to pluripotency. This is, of course, highly desirable because we want to eliminate any defective cells from the derived hiPSC lines; however, if p53 arrests the cell cycle in response to the detection of DNA damage, it is quite possible that the cell also 
tries to repair the damage. Furthermore, we know that genomewide active DNA demethylation is required to reprogramme the genome for totipotency in primordial germ cells (15) and that this process is dependent upon base-excision DNA repair. Some of the original studies of the impact of a p53 knockout were performed using Terc-/- mice with critically short telomeres, a condition that would be expected to trigger a DNA damage response; however, this is a rather exceptional case because we would not expect to find a significant number of somatic cells with such short telomeres in human samples collected for iPSC generation. Studying the impact of DNA repair mechanisms on the reprogramming process would be better achieved by generating hiPSCs from individuals suffering from diseases that result from DNA repair deficiencies.

\section{GENERATION OF HIPSCS FROM DNA REPAIR- DEFICIENT SOMATIC CELLS \\ Overview of DNA repair pathways and genes that lead to DNA repair diseases}

Several repair systems can be activated in response to a range of lesions that affect DNA. DNA double-strand breaks (DSBs) are one of the most severe forms of DNA damage, and can lead to cell death, genomic instability or oncogenic transformations if unrepaired or mis-repaired (see Figure 2). Two key mechanisms exist to repair this type of lesion, namely, the homologous recombination (HR) or non-homologous end-joining (NHEJ) pathways. The former is considered to be less error prone because it relies upon the presence of a DNA template (usually the other allele of a mutated gene) to regenerate the sequence of DNA bases around the site of the DSB. NHEJ ligates the broken DNA strands directly using short regions of homology to guide the repair. If these regions of "microhomology" are not accurate, NHEJ will still function but is imprecise, and the improperly matched nucleotides are eliminated, which can lead to mutation and/or chromosome damage.

The protein complexes that mediate HR or NHEJ comprise several proteins. For example, NHEJ-mediated DSB repair is initiated by the binding of the Ku protein to dsDNA ends, followed by recruitment of the catalytic subunit of the DNA-dependent protein kinase (DNA-PKcs), end processing and recruitment of the ligation complex comprised of DNA Ligase IV, XRCC4 and XLF, which performs the final ligation step. Mutations occurring in the genes that encode these proteins or the polypeptides from which they are constructed affect the functionality of the NHEJ and HR pathways and are known to result in a number of diseases. Not surprisingly, most of these conditions are associated with an increased predisposition to cancer, but there are a number of other developmental and cellular deficits specific to each disease. Several studies have generated hiPSCs that may be used to model the individual diseases in vitro. Single-strand breaks occur more frequently (16), but the repair systems associated with these lesions are very effective. Although other DNA repair systems include mismatch repair and nucleotide excision repair, few data are available concerning the impact of these repair mechanisms on hiPSC generation and survival. Therefore, we will focus on the mutations affecting DSB repair in this review.

\section{HIPSC MODELS OF DNA REPAIR DEFICIENCIES Fanconi anaemia}

Fanconi anaemia is a BM failure syndrome characterised by developmental abnormalities, an enhanced pre-disposition to cancer and the progressive loss of haematopoietic cells; the disease is usually fatal before the age of 40. Fifteen genes (also known as complementation groups) contribute to this disease, including FANCA, FANCB, FANCC, FANCD1 (BRCA2), FANCD2, FANCE, FANCF, FANCG, FANCL, FANCI, FANCJ, FANCM, FANCN and FANCP $(17,18)$, but the exact mechanisms by which mutations in these genes can lead to BM failure have not been confirmed. Recent studies in the field have established the existence of an FA pathway in which eight of the FA proteins, namely, FANCA, -B, -C, -E, $-F,-G,-L$ and $-M$, form a nuclear complex that is thought to function as a ubiquitin ligase in response to DNA damage or DNA replication stress. In response to stress, this multimeric FA core complex monoubiquinates FANCD2 and FANCI, which then recruit other downstream FA proteins, FANCD1/ BRCA2, FANCJ, and FANCN, to sites of DNA damage. Once the monoubiquitinated FANCD2 and its associated partners (FANCI, FANCD1, FANCJ, and FANCN) are correctly localised on the sites of DNA damage, they associate with other DNA repair proteins, such as NBS1, RAD51 and BRAC1, to initiate the DNA repair process (19).

Fanconi anaemia can be treated by BM transplants from HLA-identical siblings; if such individuals are not available, non-related donors can be used, but the outcomes are less favourable. The ultimate goal of many groups working on Fanconi anaemia is to develop protocols to transplant genetically corrected haematopoietic stem cells. These cells should have a selective growth advantage over those present in the patient's bone marrow and should progressively replace the affected stem cells over time. The generation of genetically corrected hiPSCs would be a useful first step in this development; however, initial studies have suggested that reprogramming the dermal fibroblasts directly from Fanconi anaemia patients into hiPSCs might not be possible (20). Human iPSCs were only generated from fibroblasts that had been previously corrected by stable transfection of FANCA or FANCD2, suggesting that the DNA repair / chromosome stability function mediated by the Fanconi core complex is important for reprogramming. Subsequent studies have shown that hiPSCs can be generated from FANCA and FANCC fibroblasts, though the efficiency of this process is much lower (21) and is feasible only under hypoxic conditions. These data highlight the probable involvement of the Fanconi anaemia repair pathway in hiPSC generation. The pathway activation that results from cellular stress during reprogramming increases the expression levels 


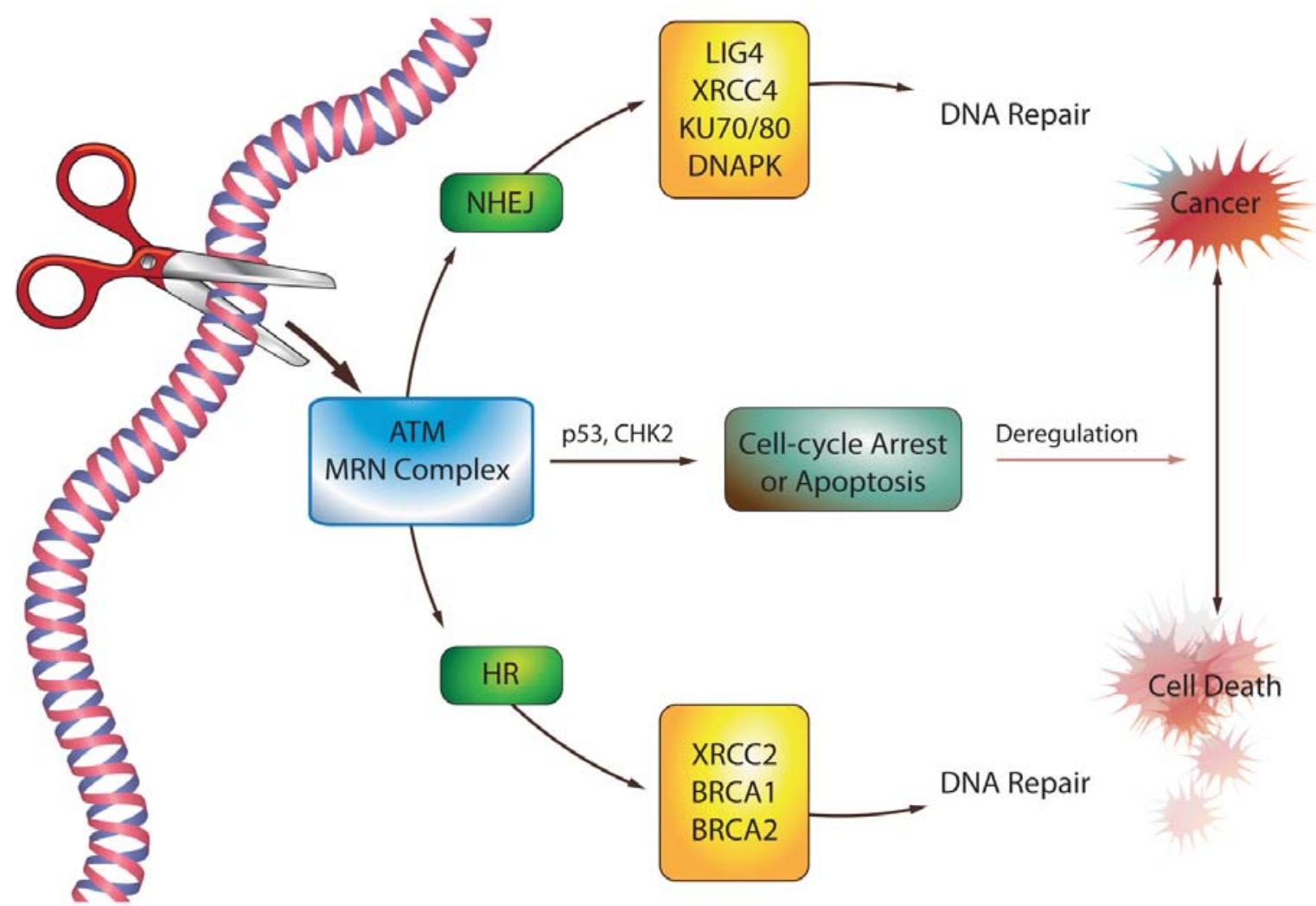

Figure 2. Schematic overview of two key mechanisms used by mammalian cells to repair DSBs.

of p53, p21 and p19 in both wildtype and Fanconi-patient fibroblasts. Interestingly, the number of DSBs (indicated by $\gamma$-H2A.X foci) increases during reprogramming, even in wildtype cells, suggesting a possible means of activating the Fanconi pathway. However, this result requires cautious interpretation because pluripotent cells show higher levels of $\gamma$-H2A.X foci than their differentiated counterparts, which may be related to a $\gamma$-H2A.X function unrelated to DNA repair (22). Even before reprogramming, Fanca-- fibroblasts have a higher level of $\gamma$-H2A.X foci, DSBs and chromosomal abnormalities; thus, it is possible that the low reprogramming efficiency may simply arise because a large number of cells are eliminated (by apoptosis) during the reprogramming process because they exceed a given threshold level of DNA damage. Work performed in our labs shows that hiPSCs can also been generated from $\mathrm{FANCC}^{-1-}$ fibroblasts (23) under normoxic conditions. These hiPSCs express the typical pluripotency marker genes and demonstrate robust multi-lineage differentiation in vitro (embryoid bodies); however, they produce abnormal teratomas consisting almost primarily of immune cells, indicating deficiencies in their in vivo differentiation potential. The levels of karyotypic abnormalities present in the FANCC ${ }^{-1}$ hiPSCs were significantly higher than in the parent fibroblasts. As a comparison, the stable knockdown of the FANCC gene by shRNA in hESCs showed a relatively stable karyotype, indicating that the additional chromosomal abnormalities may be a consequence of the hiPSC reprogramming process. In terms of enhancing our ability to model Fanconi anaemia in vitro, the FANCC ${ }^{-1-}$ hiPSCs demonstrated a greatly reduced ability to form haematopoietic colonies in methylcellulose plus haematopoietic growth factors. Although committed haematopoietic progenitors did form and proliferated at similar rates to those derived from wildtype hiPSCs, the numbers of such cells destroyed by apoptosis was much greater in the FANCC ${ }^{-1}$ hiPSCs. These combined data indicate that the activation of the Fanconi pathway is indispensable for cellular reprogramming, the maintenance of genomic stability and the survival of embryonic haematopoietic progenitors.

\section{MUTATIONS IN THE NHEJ PATHWAY - XLF AND LIGASE IV}

Although the derivation of hiPSCs from cells with mutations in Fanconi pathway genes has indicated some aspects of the impact of DNA repair mechanisms on reprogramming, there are other diseases affecting different pathways that may help to shed more light on the subject. Two genes in this pathway, Cernunnos (also known as XLF) and DNA ligase IV, are important for the final ligation step to re-join the two sides of the double-strand break. Mutations in these genes are known to cause diseases. Cernunnosdeficiency syndrome is a rare recessive autosomal disorder caused by mutations in the XLF gene, a key factor involved in the end-joining step of the DNA non-homologous end- 
joining (NHEJ) process. In humans, this disease manifests as severe immunodeficiency coupled to developmental defects (microcephaly and growth delays) (24). At the cellular level, the inhibition of NHEJ produces higher levels of unrepaired DSBs, but the consequence of this for haematopoietic differentiation appears to be similar to that observed for the Fanconi pathway in that haematopoietic progenitors do arise but are subjected to apoptotic attrition. Human iPSCs carrying the C169T (R57X) mutation in the XLF gene were also morphologically similar to unaffected control iPSCs but were unable to form teratomas following injection into immune-compromised mice (25). Defects have been observed in other differentiated cell types derived from XLF-deficient iPSCs; for example, neuronal-like cells showed differences in the expression patterns of the microtubule protein betaIII-tubulin, suggesting an altered cytoskeletal structure. The reduced NHEJ activity in XLF-deficient hiPSCs was demonstrated using fluorescent reporter constructs of NHEJ and HR activity (26). However, this analysis also indicated that the cells attempt to compensate for the reduction in NHEJ activity by increasing HR, suggesting some degree of crosstalk between the two methods for repairing double-strand breaks. Despite this, XLF-deficient hiPSCs were unable to repair DSBs efficiently after exposure to ionising radiation and showed a much higher percentage of cells with unrepaired double-strand breaks under normal conditions. This result suggests that NHEJ is required to repair the damage induced by the normal culture of hiPSCs.

Patients with LIG4 deficiency (OMIM \# 606593) show a range of phenotypic abnormalities $(27,28)$. Most of the mutations identified to date impact the ability of DNA Ligase IV to interact with its partner, XRCC4, or disrupt the ligase catalytic domain. However, the precise nature of the mutations determine the level of Ligase IV activity present in the cell, thus the disease is subject to wide variation in humans. We have been able to generate hiPSCs from Ligase IV-deficient patients, which, in parallel with the other DNA repair defects mentioned thus far, are morphologically indistinguishable from control hiPSCs (29). As expected, the efficiency of derivation is low, though the predicted karyotypic instability of Ligase IV iPSCs manifests in an unusual manner. Tilgner et al reported that hiPSCs with an apparently normal karyotype could be derived from patient fibroblasts with pronounced karyotypic abnormalities, whereas the hiPSCs derived from patient fibroblasts with a normal karyotype were prone to the accumulation of chromosomal abnormalities. The low reprogramming efficiency and karyotypic abnormalities observed for Ligase IV hiPSCs suggest that NHEJ-mediated DSB repair is important for both cellular reprogramming and the maintenance of genomic stability in hiPSCs. Similar to the hiPSCs derived from FA and XLF patients, Ligase IV patient-specific hiPSCs show in vitro and in vivo deficiency in differentiation capacity, the inability to repair DSBs and enhanced apoptosis (29). Although all the abovementioned patient-specific hiPSC lines are able to undergo differentiation to blood progenitor cells, the proliferating subset of those undergo apoptosis, resulting in a reduced number of committed blood progenitor cells, which is most likely the underlying cause of the pancytopaenia observed frequently in these patients early in childhood. Together, these data suggests that the proper functioning of NHEJ-mediated DSB repair is important for the cellular reprogramming of differentiated cells to hiPSCs, for the maintenance of their genomic stability and for the survival of proliferating blood progenitors.

\section{CONCLUDING REMARKS}

The generation of hiPSCs from somatic cells with DNA repair defects is still in its infancy, but the fact that cellular reprogramming works, albeit at a much lower efficiency, for cells with the possible accumulation of unrepaired DNA damage encourages the belief that in vitro models of DNA repair diseases can be created. This activity should be of great value in furthering our understanding of the consequences of DNA repair defects for embryonic development and the long-term survival of adult, tissuespecific stem cells and their genomic stability. A note of caution needs to be exercised if the hiPSCs are to be used for future cell-replacement therapies. Our work and that of others has shown that both the fibroblasts and hiPSCs derived from patients with mutations in DNA damage repair genes show a great degree of chromosomal abnormalities. It is, therefore, logical to screen primary human fibroblasts as early as possible for the presence of chromosomal abnormalities. Given that DNA damage repair proteins are needed during the cellular reprogramming process, it is imperative to perform gene correction in fibroblasts prior to reprogramming. The differentiation of genetically corrected hiPSC lines from such patients should then provide the functional and necessary target cells needed for the treatment of their conditions.

\section{References}

1. Hyslop LA, Stojkovic M and Lako M. Human embryonic stem cell biology and clinical implications. Expert reviews in Molecular Medicine 2005; 7:-21

2. Takahashi K, Okita K, Nakagawa M, Yamanaka S. Induction of pluripotent stem cells from fibroblast cultures. Nat Protoc. 2007; 2(12):3081-9.

3. Yamanaka S.Induction of pluripotent stem cells from mouse fibroblasts by four transcription factors. Cell Prolif. 41 Suppl 2008; 1:51-6

4. Ohnuki M, Takahashi K, Yamanaka S. Generation and characterization of human induced pluripotent stem cells. Curr Protoc Stem Cell Biol. Chapter 4:Unit 4A.2 2009;

5. Saretzki G, Armstrong L, Leake A, Lako M, von Zglinicki T. Stress defense in murine embryonic stem cells is superior to that of various differentiated murine cells. Stem Cells. 2004; 22(6):962-71 
6. Saretzki G, Walter T, Atkinson S, Passos JF, Bareth B, Keith WN, Stewart R, Hoare S, Stojkovic M, Armstrong L, von Zglinicki T, Lako M. Downregulation of multiple stress defense mechanisms during differentiation of human embryonic stem cells. Stem Cells. 2008; 26(2):455-64

7. Armstrong L, Tilgner K, Saretzki G, Atkinson SP, Stojkovic M, Moreno R, Przyborski S, Lako M. Human induced pluripotent stem cell lines show stress defense mechanisms and mitochondrial regulation similar to those of human embryonic stem cells. Stem Cells 2010; 28(4):661-73.

8. Kenyon J, Gerson SL The role of DNA damage repair in aging of adult stem cells, Nucleic Acids Res. 2007; 35: 7557-7565.

9. Nouspikel T, Hanawalt PC Terminally differentiated human neurons repair transcribed genes but display attenuated global DNA repair and modulation of repair gene expression, Mol. Cell. Biol. 2000; 20: 1562-1570.

10. Momcilovic O, Knobloch L, Fornsaglio J, Varum S, Easley $\mathrm{C}$, Schatten G DNA damage responses in human induced pluripotent stem cells and embryonic stem cells, 2010; PLoS ONE 5: e13410.

11. Mathur D, Danford TW, Boyer LA, Young RA, Gifford DK, Jaenisch R. Analysis of the mouse embryonic stem cell regulatory networks obtained by ChIP-chip and ChIP-PET. Genome Biol. 2008; 9(8):R126

12. Fong YW, Inouye $C$, Yamaguchi $T$, Cattoglio $C$, Grubisic I, Tjian R. A DNA repair complex functions as an Oct4/Sox2 coactivator in embryonic stem cells. Cell. 2011; 147(1):120-31

13. Zhan Q, Carrier F, Fornace AJ Jr. Induction of cellular p53 activity by DNA-damaging agents and growth arrest. Mol Cell Biol. 1993; 13(7):4242-50.

14. Marión RM, Strati K, Li H, Murga M, Blanco R, Ortega S, Fernandez-Capetillo O, Serrano M, Blasco MA. A p53-mediated DNA damage response limits reprogramming to ensure iPS cell genomic integrity. Nature. 2009; 460(7259):1149-53

15. Hajkova P, Jeffries SJ, Lee C, Miller N, Jackson SP, Surani MA. Genome-wide reprogramming in the mouse germ line entails the base excision repair pathway. Science. 2010; 329(5987):78-82

16. Caldecott KW Single-strand break repair and genetic disease. Nat Rev Genet. 2008; 9(8):619-31

17. Niedernhofer LJ, Lalai AS, Hoeijmakers JH. Fanconi anemia (cross)linked to DNA repair. Cell.2005; 123(7):1191-1198.

18. Kim Y, Lach FP, Desetty R, Hanenberg H, Auerbach AD, Smogorzewska A.Mutations of the SLX4 gene in Fanconi anemia. Nat Genet. 2011; 43(2):142-146.

19. Auerbach AD, Wolman SR. Susceptibility of Fanconi's anaemia fibroblasts to chromosome damage by carcinogens. Nature. 1976; 261(5560):494-6.

20. Raya A, Rodríguez-Pizà I, Guenechea G, Vassena R, Navarro S, Barrero MJ, Consiglio A, Castellà M, Río P,
Sleep E, González F, Tiscornia G, Garreta E, Aasen T, Veiga A, Verma IM, Surrallés J, Bueren J, Izpisúa Belmonte JC. Disease-corrected haematopoietic progenitors from Fanconi anaemia induced pluripotent stem cells. Nature. 2009; 460(7251):53-9.

21. Müller LU, Milsom MD, Harris CE, Vyas R, Brumme KM, Parmar K, Moreau LA, Schambach A, Park IH, London WB, Strait K, Schlaeger T, Devine AL, Grassman E, D'Andrea A, Daley GQ, Williams DA. Overcoming reprogramming resistance of Fanconi anemia cells. Blood. 2012; 119(23):5449-57

22. Turinetto V, Orlando L, Sanchez-Ripoll Y, Kumpfmueller B, Storm MP, Porcedda P, Minieri V, Saviozzi S, Accomasso L, Cibrario Rocchietti E, Moorwood K, Circosta P, Cignetti A, Welham MJ, Giachino C. High basal $\gamma \mathrm{H} 2 \mathrm{AX}$ levels sustain self-renewal of mouse embryonic and induced pluripotent stem cells. Stem Cells. 2012; 30(7):1414-23.

23. Yung SK, Tilgner K, Ledran MH, Habibollah S, Neganova I, Singhapol C, Saretzki G, Stojkovic M, Armstrong L, Przyborski S, Lako M Human pluripotent stem cell models of Fanconi Anaemia deficiency reveal animportant role for Fanconi Anaemia proteins in cellular reprogramming and survival of haematopoietic progenitors. Stem Cells (in press).2012;

24. Buck D, Malivert L, de Chasseval R, et al Cernunnos, a novel nonhomologous end-joining factor, is mutated in human immunodeficiency with microcephaly. Cell. 2006; 124(2):287-99.

25. Tilgner K, Neganova I, Singhapol C, Saretzki G, Evans J, Gorbunova V, Gennery A, Przyborski S, Stojkovic M, Armstrong L, Jeggo P and Lako M. A human induced pluripotent stem cell model of Cernunnos deficiency reveals an important role for XLF in the survival of most primitive haematopoietic Progenitors. (under review with Stem Cells) 2012;

26. Seluanov A, Mao Z, Gorbunova V. Analysis of DNA double-strand break (DSB) repair in mammalian cells. J. Vis. Exp. 2012; 43:pii:2002.

27. O’Driscoll M, Cerosaletti KM, Girard PM, Dai Y, Stumm M, Kysela B, et al.DNA ligase IV mutations identified in patients exhibiting developmental delay and immunodeficiency. Mol Cell 2001; 8(6): 1175-1185

28. Riballo E, Critchlow SE, Teo SH, Doherty AJ, Priestley A, Broughton B, et al Identification of a defect in DNA ligase IV in a radiosensitive leukaemia patient. Curr Biol. 1999; 9(13): 699-702

29. Tilgner K, Neganova I, Yung S, Singhapol C, Saretzki G, Evans J, Gorbunova V, Gennery A, Przyborski S, Stojkovic M, Armstrong L, Jeggo P and Lako M A human iPSC model of Ligase IV deficiency reveals an important role for NHEJ-mediated-DSB repair in the survival and genomic stability of induced pluripotent stem cells and emerging haematopoietic progenitors. Cell Death and Differentiation (under final review).2012; 